\title{
Photopolymerization with Light Fields Possessing Orbital Angular Momentum: Generation of Helical Microfibers
}

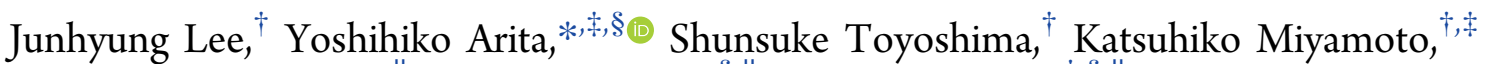

Paris Panagiotopoulos, " Ewan M. Wright, ${ }^{\S, \|}$ Kishan Dholakia, ${ }^{\dagger, \S, \|}$ and Takashige Omatsu*, ${ }^{, \dagger, \$}$

${ }^{\dagger}$ Graduate School of Engineering, Chiba University, 1-33 Yayoi-cho, Inage-ku, Chiba-shi 263-0022, Japan

${ }^{\ddagger}$ Molecular Chirality Research Center, Chiba University, 1-33 Yayoi-cho, Inage-ku, Chiba-shi 263-0022, Japan

${ }^{\S}$ SUPA, School of Physics and Astronomy, University of St Andrews, North Haugh, Fife KY16 9SS, United Kingdom

"College of Optical Sciences, The University of Arizona, 1630 East University Boulevard, Tucson, Arizona 85721-0094, United States

Supporting Information

\begin{abstract}
Photopolymerization is a powerful technique to create arbitrary micro-objects with a high spatial resolution. Importantly, to date all photopolymerization studies have been performed with incident light fields with planar wavefronts and have solely exploited the intensity profile of the incident beam. We investigate photopolymerization with light fields possessing orbital angular momentum, characterized by the topological charge $l$. We show both experimentally and theoretically that, as a consequence of nonlinear self-focusing of the optical field, photopolymerization creates an annularshaped vortex-soliton and an associated optical fiber, which breaks up into $|l|$ solitons or microfibers. These microfibers exhibit helical trajectories with a chirality determined by the sign of $l$ due to the orbital angular momentum of the light field and form a bundle of helical-microfibers. This research opens up a new application for light fields with orbital angular momentum, and our generated microfibers may have applications in optical communications and micromanipulation.
\end{abstract}

KEYWORDS: microstructured fibers, optical vortices, polymer waveguides, micro-optical devices

$\mathrm{P}$ hotopolymerization, the process of using ultraviolet (UV) light to activate polymerization within resins, is a powerful approach to create arbitrary, transparent micro-objects with a resolution below the diffraction limit. ${ }^{1}$ Such microstructures have been optimized for optical manipulation ${ }^{2,3}$ and are finding application elsewhere, including micro-optics, ${ }^{4,5}$ mechanical microstructures, ${ }^{6}$ and polymer crystallography. ${ }^{7}$ Furthermore, due to self-focusing and self-trapping, the polymerized material can form a waveguide when irradiated by a Gaussian beam (the so-called laser-induced self-written waveguide or LISW), which develops into a single-induced fiber, that can be as long as millimeters in length. ${ }^{8-10}$ Importantly, to date all photopolymerization studies have been performed with incident light fields possessing planar wavefronts. A few studies have utilized optical vortex beams, which carry helical phase fronts possessing orbital angular momentum (OAM), to fabricate annular profiles. ${ }^{11,12}$ Crucially, however, these techniques are yet to exploit more complex forms of incident beam wavefronts going beyond the standard planar wavefront form.

The origin of OAM is inherently linked to the local direction of the Poynting vector, which denotes the direction of the energy flux density. Considering any point in the beam, the Poynting vector is perpendicular to the helical phase front and hence possesses an azimuthal momentum component. Such fields have a characteristic $\exp ( \pm i l \phi)$ phase variation with $\phi$ the azimuthal angle in cylindrical coordinates, and may be described by Laguerre-Gaussian (LG) transverse modes. ${ }^{13}$ The topological charge or azimuthal index $l$ of the beam denotes the integer multiple of $2 \pi$ that the field phase accumulates upon circling the beam center. OAM has generated immense interest over the last two decades and led to a diverse range of applications. Examples include studies in optical manipulation of both mesoscopic particles ${ }^{14,15}$ and quantum gases, ${ }^{16}$ micromachining, ${ }^{17-19}$ and quantum information processing. $^{20,21}$

In this Article, we investigate photopolymerization with an incident optical vortex and show for the first time that using such light fields that possess OAM for photopolymerization leads to "spiraling" of the generated polymerized fibers, with a chirality determined by the sign of $l$ (see Figure 1 for schematic representation). In particular, due to a transverse modulation instability in the nonlinear self-focusing photopolymer, the vortex beam breaks up into the $|l|$ solitons or

Received: July 13, 2018

Published: October 8, 2018 

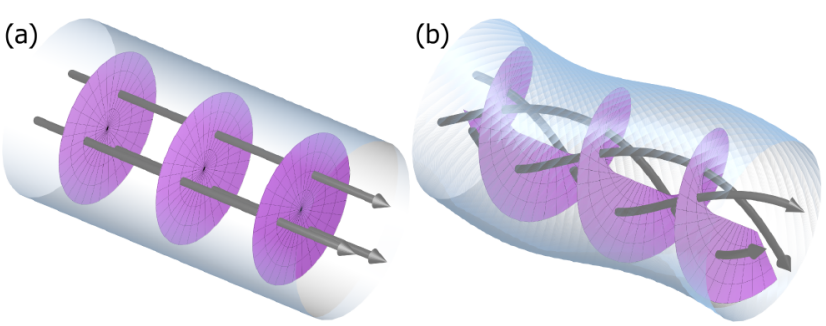

Figure 1. Schematic of a beam propagating through a light-curing resin, which is photopolymerized into optical fibers due to selffocusing and self-trapping. (a) Planar wave propagating in the photopolymerized fiber, which is straight and parallel to the beam axis. (b) A vortex beam with a helical wavefront photopolymerizing the resin, which evolves into $|l|$ helical fibers. The arrows indicate the Poynting vectors or the energy flow relative to their wavefronts.

microfibers, each of which exhibit helical trajectories and together form a bundle of helical microfibers. This therefore represents a new physical manifestation of the use of OAM light fields. Our numerical simulations, based on the nonlinear paraxial wave equation for the photopolymer, captures all the experimental observations for a variety of optical vortices characterized by $l$. In a broader context, our work adds a new facet to the emergent field of helical fibers that have themselves recently come to the fore in the photonic crystal community as a route to generating fields with OAM. ${ }^{22,23}$

\section{RESULTS AND DISCUSSION}

UV curing resins are a mixture of photoinitiator, monomer and oligomer molecules. Upon irradiation of UV light, the photoinitiators are excited then emit electrons to become radicals, which are highly reactive to the monomers and oligomers. Consequently photopolymerization takes place with a time lag of about $0.1 \mathrm{~s}$ and induces a permanent refractive index change of $\Delta n \approx 0.04$ from $n_{\text {unc }}=1.52$ to $n_{\text {cur }}=1.56$ at $405 \mathrm{~nm}$ for the resin used in this experiment (see the Materials and Methods for the experimental details). As a result, the incident optical beams are self-focused and self-trapped upon photopolymerization. $^{24}$

Gaussian Beam with $\boldsymbol{I}=\mathbf{0}$. Figure 2a shows time-lapse images of the photopolymerization process induced by irradiation of a Gaussian beam with $l=0$. The beam is focused (depicted as two arrows) at the glass/resin boundary (indicated as dashed lines) starting at time $t=0 \mathrm{~s}$. The resin is continuously irradiated for $0.9 \mathrm{~s}$ while recording the photopolymerization process by cameras CMOS1 and CMOS2 for side and axial views (see Materials and Methods for details). It is evident that the beam propagating in the photopolymer is self-focusing and self-trapping with the formation of a singleinduced fiber with a length $>150 \mu \mathrm{m}$ (see also Supporting Information, video 1 ).

After photopolymerization, the glass substrate with the polymerized structure is rinsed with acetone and is dried for SEM imaging. Figures $3 a-c$ show SEM images of the photopolymerized structure produced by irradiation of a Gaussian beam with $l=0$. A cylindrically symmetric fiber is formed with a length of $160 \mu \mathrm{m}$, where the diameter of the fiber decreases from $d_{\text {ini }}=2.5 \mu \mathrm{m}$ at the glass $/$ polymer interface to $d_{\text {end }}=0.9 \mu \mathrm{m}$.

LG Beams with $|/|=1$. LG beams carrying OAM with topological charge $l= \pm 1$ also form single fiber structures but with broken cylindrical symmetry along the beam propagation direction (see Figure $2 \mathrm{~b}$ and the associated Supporting Information, video 2). Most intriguingly, its rotation is evident in the axial view recorded in Supporting Information, video 2. Figure $3 \mathrm{~g}$ shows a helical-fiber with its chirality (clockwise) set by the LG beam with $l=1$. Two parts of the twisted features observed are indicated by the enclosed dashed-line squares and are expanded in Figures 3h,i. Importantly, the opposite handedness of the LG beam with $l=-1$ creates a helicalfiber with counterclockwise rotation (see Figures $3 d-f$ ). This is the first direct demonstration that photopolymerization is itself spiraling in line with the nature of the spiraling wavefronts present within an incident beam. We note that
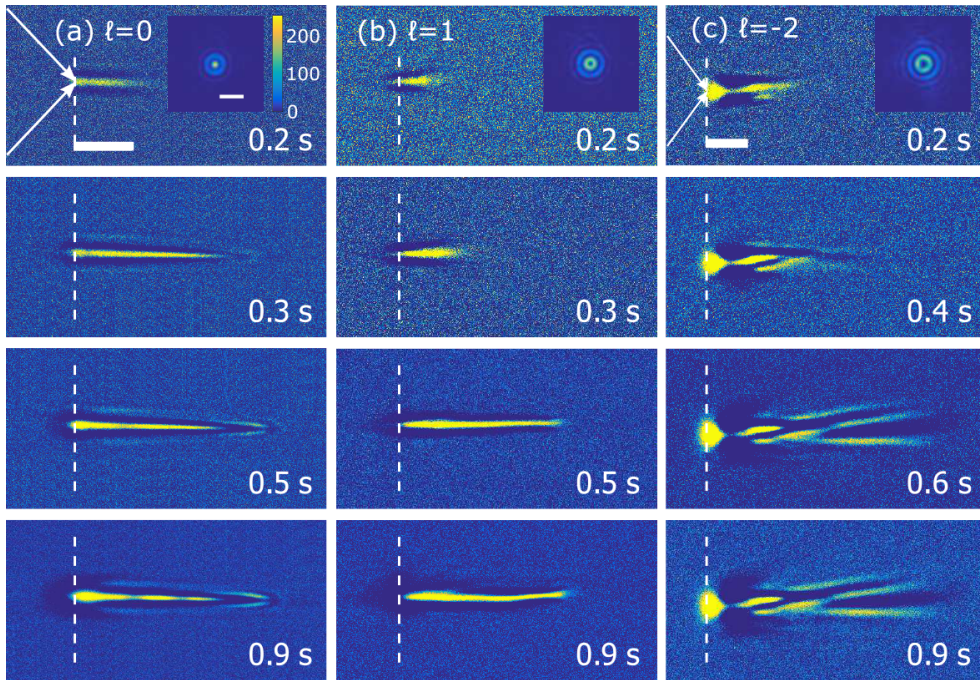
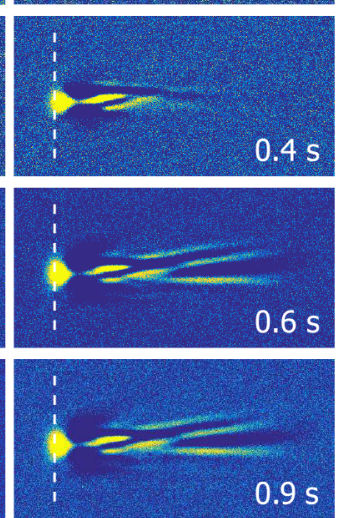
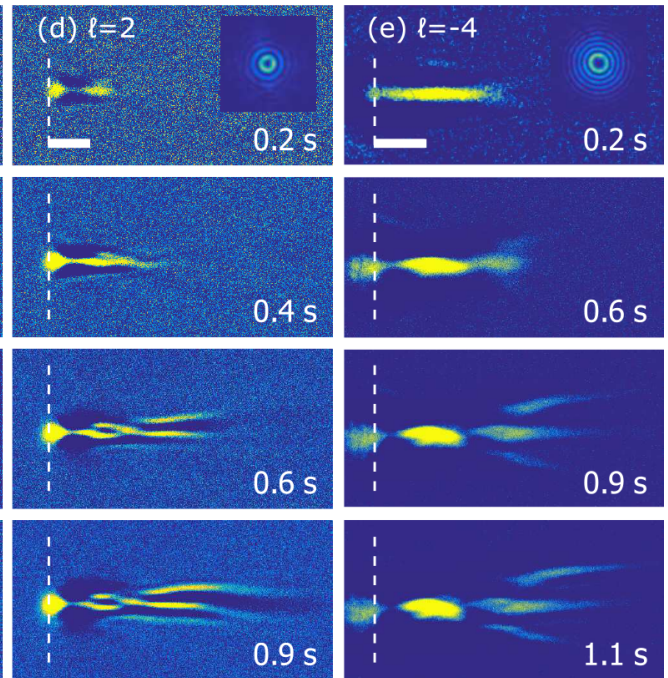

Figure 2. Time-lapse images of the photopolymerization process. (a) Gaussian beam with $l=0$ (see Supporting Information, video 1 for side-/ axial-views). LG beams with (b) $l=1$ (Supporting Information, video 2 for side-/axial-views), (c) $l=-2$, (d) $l=2$ (Supporting Information, video 3 for side-views of $|l|=2$ ), (e) $l=4$. White arrows indicate the beam focused at the glass/resin boundary (indicated as dashed lines). The scale bar shows $50 \mu \mathrm{m}$ and applies to all figure panels. Beam exposure starts at time $t=0 \mathrm{~s}$ and ends at $t=0.9 \mathrm{~s}$ with an optical power of $3 \mathrm{~mW}$. Inset panels show focused beam profiles projected onto the glass surface for each topological charge $l=0,1, \mp 2,-4$ where the beam waist radius is $0.35,0.50,0.63$, and $0.79 \mu \mathrm{m}$, respectively. The scale bar (inset) indicates $2 \mu \mathrm{m}$ and applies to all inset panels. 

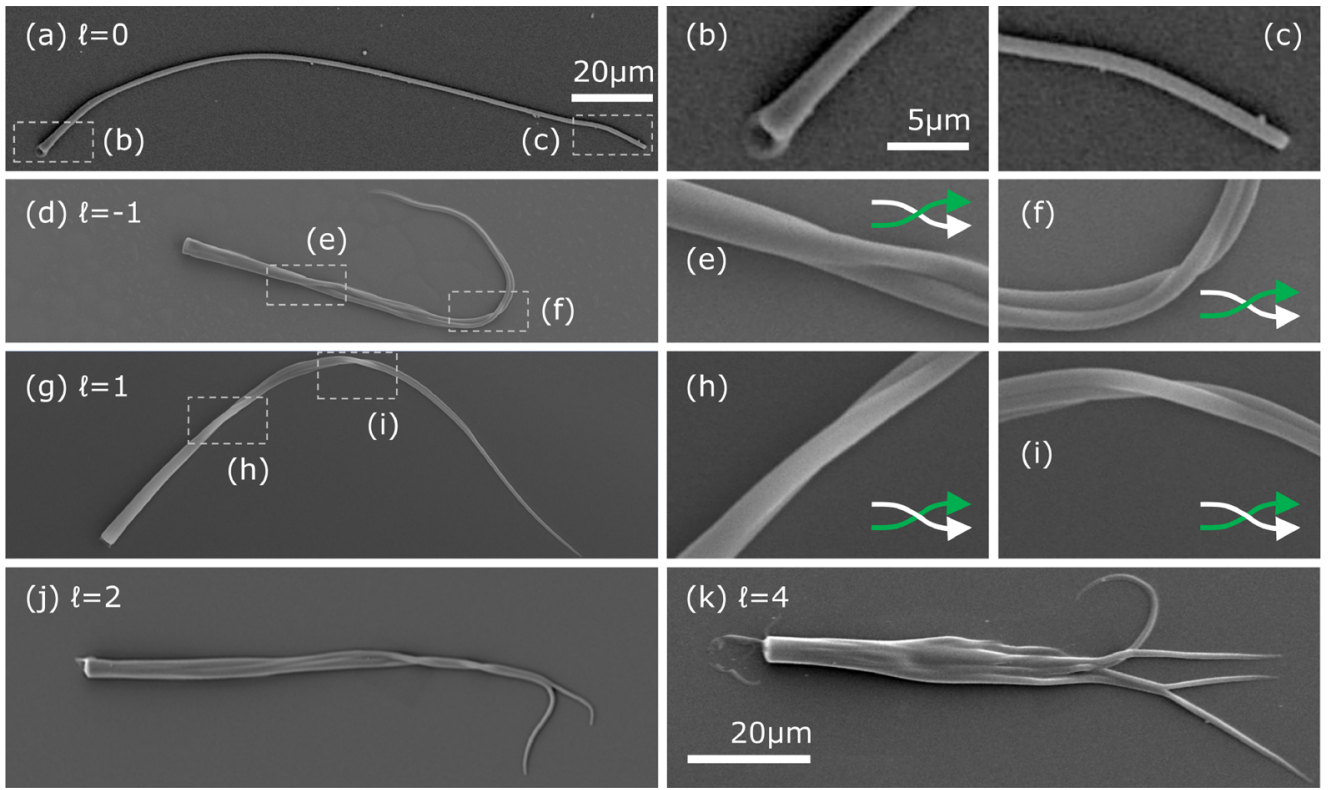

Figure 3. SEM images of the photopolymerized fiber structures created by laser irradiation of a Gaussian beam with $l=0$ and LG beams with $|l| \geq 1$. (a) $l=0$. (b, c) Expanded views of the selected areas in (a) showing the cylindrical structures. (d) $l=-1$. (e, f) Expanded views of the selected areas in $(\mathrm{d})$ showing the counterclockwise helix. $(\mathrm{g}) l=1$. (h, i) Expanded views of the selected areas in $(\mathrm{g})$ showing the clockwise helix. (j) $l=2$ exhibiting two fiber branches. (k) $l=4$ exhibiting four branches. The $20 \mu \mathrm{m}$ scale bar in panel (a) applies to (d), (g), and (j), while the $5 \mu \mathrm{m}$ bar in panel (b) applies to (c), (e), (f), (h), and (i). Experimental parameters are the same as in Figure 2.

the spiraling structure in this case still constitutes one fiber that is not breaking apart (see Figures $2 \mathrm{~b}$ and $3 \mathrm{~d}, \mathrm{~g}$ ).

Higher Order LG Beams with $I / \mid \geq 2$. Figures $2 \mathrm{c}, \mathrm{d}$ show time-lapse images of growing fibers by LG beams with $l= \pm 2$ (see also Supporting Information, video 3 for the side views of $|z|=2)$. The polymerized fiber is split into two microfibers, which are coiled around each other either counterclockwise $(l=-2)$ or clockwise $(l=2)$ along the beam propagation axis. We further investigate the case of an LG beam with $l=4$ with an annular beam diameter $\geq 2 \mu \mathrm{m}$ focused at the glass/ resin interface (see Figure 2e). The time-lapse images reveal that the polymerized fiber exhibits at least three branches (with potentially a fourth out-of-focus and invisible) of microfibers. Figures 3j,k show the SEM images of the fibers polymerized by the vortex beams with $l=2$ and $l=4$, which exhibit the corresponding number of branches. We note that this is consistent with photopolymerization with $l=3$ showing three microfibers (see Supporting Information, Figure S1). For the case of $l=|2|$, it is evident that the two microfibers are attracted each other and form a double helix with chirality determined by the sign of $l$. We note that a photopolymerized fiber produced by an LG beam with $|l| \geq 1$ exhibits a hollowcore structure with a short beam exposure time of $<0.2 \mathrm{~s}$ (see Supporting Information, Figure S2). However, for a long exposure time, the hollow center of the fiber will be polymerized because the optical field extends into the uncured photopolymer.

Spatial Profile of Microfibers and V-Value. In order to gain further insight into photopolymerization with an optical vortex, we have characterized the spatial profile of the polymerized fibers fabricated by different LG modes $(|l| \leq 4)$. Figure $4 \mathrm{a}$ shows the fiber diameter as a function of distance from the initial curing facet towards the fiber end based on the SEM images. The initial diameter of the fiber scales with the annular beam diameter at the focus, which is dependent on $\sqrt{|l|+1}$ (see Supporting Information, Figure
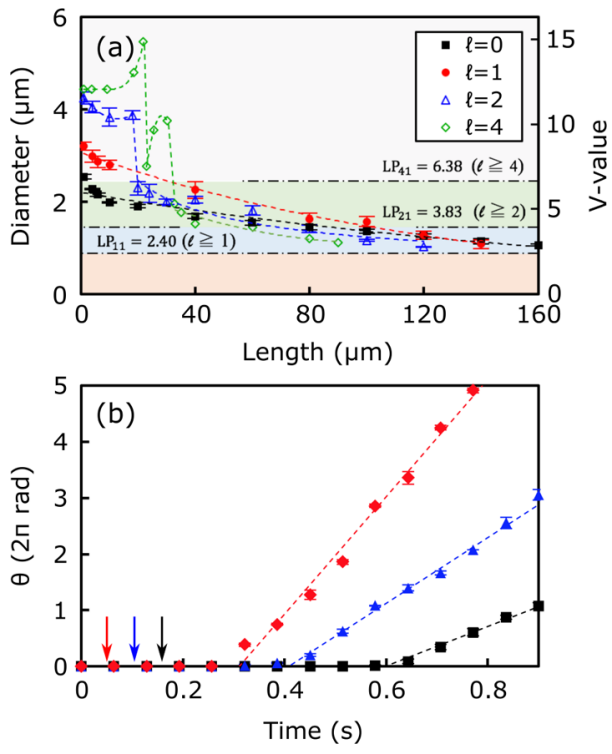

Figure 4. Fiber dimensions for different $l$ and rotation rate at different $P$. (a) Spatial profile of the fibers photopolymerized by LG beams with $|z| \leq 4$. Fiber diameter and the corresponding $V$-value are plotted as a function of fiber length or distance from the initial cure-facet. Error bars indicate $2 \sigma$. (b) Number of revolutions of the photopolymerized fiber as a function of time when irradiated by an LG beam with $l=1$ for different optical power levels, where error bars show $2 \sigma$ and arrows indicate the time when photopolymerization starts for each power level. The gradient of the curves yields their rotation rates, which are $3.5 \pm 0.4 \mathrm{~Hz}(2 \sigma), 5.9 \pm 0.4 \mathrm{~Hz}$, and $10.5 \pm$ $0.1 \mathrm{~Hz}$ for $P=1,2$, and $3 \mathrm{~mW}$, respectively. Experimental parameters are the same as in Figure 2 except for the optical power which is varied in the range of $1 \mathrm{~mW} \leq P \leq 3 \mathrm{~mW}$ in (b).

S3) ${ }^{25}$ As for the Gaussian beam $(l=0$, black squares in Figure $4 \mathrm{a})$ and the first order LG mode $(l=1$, red circles in Figure $4 a)$, the polymerized fiber diameter becomes gradually 
smaller towards the fiber end (see also Figures 3a,d). On the other hand, for $l=2,4$, there are transitions in fiber diameter due to the branching behavior. Notably, the minimum fiber diameter was always measured to be $d_{\text {end }} \approx 0.9 \mu \mathrm{m}$ at the end of each fiber.

A photopolymerized fiber has a higher refractive index than the uncured resin, which can be regarded as a single-core stepindex optical fiber. It is relevant to consider the fiber parameter, $V$, which determines the fraction of the optical power confined to the fiber core for a specific mode and is defined as ${ }^{26} V=(2 \pi a / \lambda) \sqrt{n_{\text {cur }}^{2}-n_{\text {unc }}^{2}}$, where $a$ denotes the radius of the fiber, $\lambda=405 \mathrm{~nm}$ is the wavelength of light, and $n_{\text {cur }}=1.56$ and $n_{\text {unc }}=1.52$ are the refractive indices of the polymer before and after curing. The linearly polarized (LP) lowest order mode $\left(\mathrm{LP}_{01}\right)$ has an intensity profile similar to that of a Gaussian beam. For single mode fibers, the fraction of optical power transmitted is the confinement factor $(0 \leq \Gamma \leq 1)$, which reaches $\Gamma \approx 0.9$ near the single-mode cutoff at $V \approx 2.405$. This means that higher order modes require higher $V$ values, which in turn requires a wider core radius of the fiber to support their propagation.

In this regard, LP modes in a step index fiber are well understood, while there is little or no direct information is available about LG modes. However, they can be approximated by the most overlapping beam profiles of the LP modes, for example, $\mathrm{LP}_{11} \approx \mathrm{LG}_{01}, \mathrm{LP}_{21} \approx \mathrm{LG}_{02}, \mathrm{LP}_{41} \approx \mathrm{LG}_{04}$, within certain limits. ${ }^{27}$ By calculating the $V$ values for those LP modes, we can estimate the "cutoff" diameters of the fiber for the corresponding LG modes. The second vertical axis in Figure $4 \mathrm{a}$ indicates the $V$ value corresponding to the fiber diameter on the first vertical axis, where the three threshold lines correspond to $\mathrm{LG}_{04}, \mathrm{LG}_{02}$, and $\mathrm{LG}_{01}$ from the top. Irrespective of the topological charge $l$, all the fibers have $d_{\text {end }} \approx 0.9 \mu \mathrm{m}$, which is larger than the cutoff diameter of $\mathrm{LP}_{11}$ or $L_{01}$ mode. As for higher order modes $L_{02}$ and $L_{04}$, the polymerized fibers are split into $|l|$ microfibers, whose diameter is similar to that of the fiber polymerized with $\mathrm{LG}_{01}$, which allows its mode propagation. It is noteworthy that the scattering of light at the cured/uncured-resin boundary and the diffusion of free radials beyond the illuminated region contribute to the increase in fiber core diameter.

We note that linear polarization may lead to a nonuniform temperature gradient around the beam focus due to polarization dependent light absorption in a resin, which in turn may affect the polymerized structure. ${ }^{28} \mathrm{~A}$ more detailed polarization study would shed further insight into the role of both the electric field enhanced heat conduction and spin angular momentum of light in the photopolymerization process and be worthy of future investigation.

As experimentally shown by Ježek et al., a zeroth order Bessel beam can create a centimeter long polymerized fiber using single photon photopolymerization. ${ }^{10}$ One could therefore use an optical vortex embedded in a Bessel beam, making it a beam of higher order, for the creation of a longer helical fiber. This would also be an area of future investigation. It is also interesting to speculate about the use of perfect vortex beams, ${ }^{15,29,30}$ where the beam radius and the radial intensity profile are both independent of $l$. Here we would likely observe the overall dimensions of the fiber to be the same for any $l$, allowing for a direct comparison between the fibers with different $l$ in terms of fiber branching.
Rotation Rate. In order to associate the OAM of $l \hbar$ per photon with the optical power, we measure the rotation rate of the polymerized fiber induced by an LG beam with $l=1$ at different optical powers in the range $P=1-3 \mathrm{~mW}$. The forward scattered light through the fiber along the beam axis allows a direct measurement of its rotation rate. Supporting Information, video 2 recorded by both CMOS1 and CMOS2 (see Materials and Methods for details) includes the axial view of the polymerized fiber with $l=1$ at a power level of $3 \mathrm{~mW}$. Figure $4 \mathrm{~b}$ shows the number of revolutions of the fiber as a function of time where $t=0 \mathrm{~s}$ denotes the start of beam irradiation. Here we observe a time lag between the application of the laser field and the appearance of the refractive-index change, as indicated by arrows for different optical power. The gradient of each curve determines the rotation rate, which linearly scales with optical power in the range of $P=1-3 \mathrm{~mW}$ within an error of $\pm 15 \%$. We stress here that the rotation rate and the associate pitch of the helix are primarily determined by the induced refractive index change of the medium and are not commensurate with the helical pitch of the vortex wavefront (see Model and Simulations for details). Furthermore, we have investigated photopolymerization with circularly polarized beams compared to the linearly polarized case (see Supporting Information, Figure S4).

\section{MODEL AND SIMULATIONS}

We employ the same model and notation for propagation of UV light of frequency $\omega$ in a curing resin as described by Kewitsch and Yariv in ref 24. Then the paraxial wave equation for the slowly varying electric field envelope $E(\mathbf{r}, t)$ propagating dominantly along the $z$-axis is

$$
\frac{\partial E}{\partial z}=\frac{1}{2 k_{\mathrm{b}}} \nabla_{T}^{2} E+i k_{0} \Delta n^{\prime}(\mathbf{r}, t) E-\frac{\alpha}{2} E
$$

where $k_{\mathrm{b}}=n_{\mathrm{b}} k_{0}, n_{\mathrm{b}}$ being the background refractive index, $k_{0}=\omega / \mathcal{c}$, and $\nabla_{T}^{2}$ is the transverse Laplacian describing beam diffraction. In this model it is assumed that the field polarization state remains fixed thereby allowing for a scalar field approach, and the electric field is scaled such that $|E|^{2}$ is an intensity. Furthermore, $\Delta n^{\prime}(\mathbf{r}, t)$ is the real part of the index response due to the UV light-induced photopolymerization

$$
\Delta n^{\prime}(\mathbf{r}, t)=\Delta n_{0}{ }^{\prime}\left(1-\exp \left[-\frac{1}{U_{0}} \int_{0}^{t-\tau}\left|E\left(\mathbf{r}, t^{\prime}\right)\right|^{2} \mathrm{~d} t^{\prime}\right]\right)
$$

where $\tau$ is the monomer radical lifetime, which dictates the time lag between the application of the laser field and the appearance of the optically induced refractive-index change, and $U_{0}$ is the critical exposure needed for photopolymerization. In contrast to ref 24 , we do not include the imaginary part of the index response but rather simply include single-photon absorption via the coefficient $\alpha$ (see below).

For the NOA63 sample considered and a UV wavelength of $405 \mathrm{~nm}$ we set $n_{\mathrm{b}}=1.52, \Delta n_{0}{ }^{\prime}=0.04$, and the lag time was estimated from measurements as $\tau=0.1 \mathrm{~s}$ (see Figure $4 \mathrm{~b}$ ). It is known that in the wavelength range $350-380 \mathrm{~nm}$ the critical exposure is around $U_{0}=4.5 \mathrm{~J} / \mathrm{cm}^{2}$. However, due to the low value of the single-photon absorption at $405 \mathrm{~nm}$ based on absorption measurements we estimate that $U_{0}=500 \mathrm{~J} / \mathrm{cm}^{2}$ under the conditions of our experiment (see Supporting Information, Figure S5). Finally, due to the smallness of the 

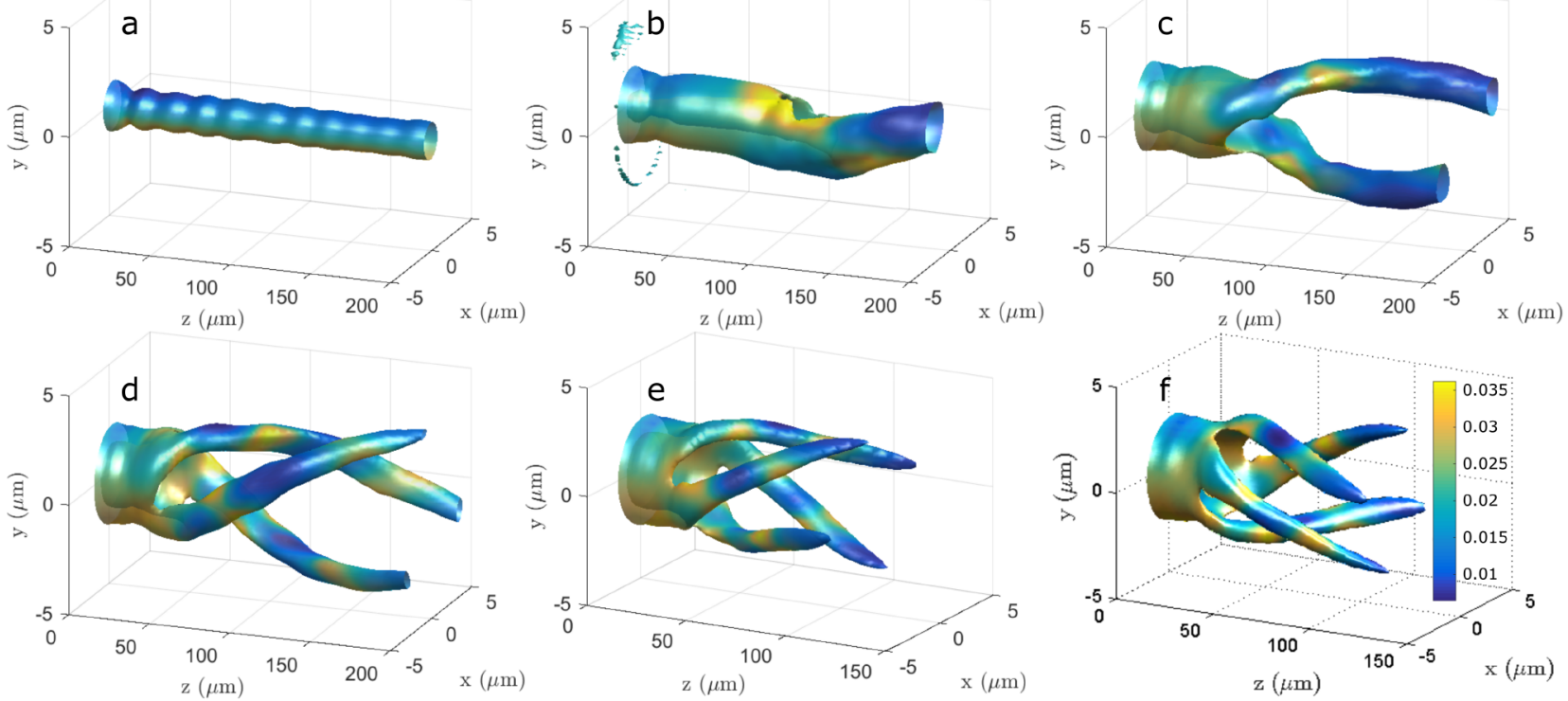

Figure 5. Isometric plots of the induced real index change $\Delta n^{\prime}(\mathbf{r}, t)$ evaluated at the exposure time $t=0.9 \mathrm{~s}$ revealing the formation of helical microfibers during the photopolymerization process: (a) Gaussian beam $(l=0),(\mathrm{b}) l=1$ (see Supporting Information, video 4 ), (c) $l=2,(\mathrm{~d})$ $l=3$ (see Supporting Information, video 5), (e) $l=4$, and (f) $l=-4$ showing the reversal of the chirality with respect to $l=4$ in plot (e).

single-photon absorption we neglect it $(\alpha=0)$ over the 200 $\mu \mathrm{m}$ propagation lengths considered.

We have performed simulations based on eqs 1 and 2 using the Beam Propagation Method for a variety of initial conditions corresponding to optical vortices characterized by their topological charge $l$. Inspection of the inset panels in Figure 2 reveals that the incident fields utilized in the experiment may be characterized as Laguerre-Gaussian (LG) beams ${ }^{31}$ with topological charge $l$, and associated radial mode index $p=(|l|+1)$, so for $l \neq 0$, the corresponding intensity profiles are composed of concentric rings of differing maximum intensities. In the numerics we have modeled the input beams as focused LG beams based on a Gaussian spot size $w_{0}=3 \mu \mathrm{m}$, which is in the range $|l| \leq 4$ gives radii of the ring of peak intensity in the range $1-2 \mu \mathrm{m}$ (see inset panels of Figure 2 ). In addition, the incident input power of $0.3 \mathrm{~mW}$ in the experiment translates to $0.1 \mathrm{~mW}$ at the sample surface and yields peak input intensities around $I_{\mathrm{p}}=1-2 \mathrm{~kW} / \mathrm{cm}^{2}$. Therefore, for the exposure time of around $1 \mathrm{~s}$ for the experiment an incident fluence of $1 \mathrm{~kJ} / \mathrm{cm}^{2}$ exceeds the critical exposure $U_{0}=0.5 \mathrm{~kJ} / \mathrm{cm}^{2}$, and the simulations are expected to display the photopolymerization process.

The results of the simulations are displayed as isometric plots of the induced real index change $\Delta n^{\prime}(\mathbf{r}, t)$ evaluated at the exposure time $t=0.9 \mathrm{~s}$, as this will reveal the formation of any permanently induced fibers during the photopolymerization process. Figure $5 \mathrm{a}$ is for $l=0$ and shows the self-focusing and self-trapping previously reported in ref 24 with the formation of a single induced fiber (and associated optical soliton) with a high degree of cylindrical symmetry around the $z$-axis throughout the propagation, see Figure $2 \mathrm{a}$ for the experimental result. In contrast, Figure $5 b$ is for $l=1$ and around $z=100 \mu \mathrm{m}$ we see that the induced fiber breaks to one side and therefore clearly breaks the cylindrical symmetry around the $z$-axis (see also Supporting Information, video 4). This behavior is similar to that seen at the bottom of panel (b) in Figure 2 for the experiment. In this context we remark that in all of the simulations a $1 \%$ amplitude noise was added to the incident LG beams to model unavoidable imperfections in the input beams, and without this added noise all of the simulations remained cylindrically symmetric: The noise was varied between $0.1-10 \%$ with similar qualitative results, though the noise was starting to overwhelm the dynamics as the upper value was approached. Finally, Figures $5 \mathrm{c}-\mathrm{e}$ show the main results of the simulations for $l=2,3,4$, and clearly support the conclusion of the experiment that helical microfibers are formed whose chirality is controlled by $l$ (see also Supporting Information, video 5 for the case of $l=3$ ). The initial vortex beams generate $|l|$ fibers that spiral around each other as also seen in the bottom plots of Figures $2 \mathrm{c}-\mathrm{e}$ for the experiment. For the case of $l=-4$, Figure $5 \mathrm{f}$ illustrates that reversing the sign of the topological charge reverses the direction of the spiraling and the chirality of the helical microfibers, compared with Figure 5e for $l=4$.

These simulations demonstrate that the main features of the experiment are captured by the model of Kewitsch and Yariv, ${ }^{24}$ and it remains to provide a physical context for the observations. Previous studies of propagation in nonlinear Kerr media or saturable nonlinear media have shown that initial vortex beams with ring intensity profiles can produce necklace beams ${ }^{32}$ composed of circumferentially arranged solitons via transverse Modulational Instabilities (MIs), and under propagation, the solitons fly off tangential to the ring, akin to free particles, as demanded by conservation of field OAM. ${ }^{33-36}$ We note that even in this case the trajectories of the emitted solitons have a sense of handedness, or chirality, see the plots in the right-hand columns of Figures 2 and 3 of ref 35, which is reversed when the sign of $l$ is changed. Such a transverse $\mathrm{MI}^{37}$ underlies the breakdown of the initial cylindrical symmetry seen in Figures $5 c-e$, where each initial vortex of topological charge $l=2,3,4$ breaks into $|l|$ fibers. Changing the noise strength modifies when the symmetry breaking sets in along the $z$-axis but the qualitative picture remains the same. However, in our case, the solitons and fibers so formed are seen to spiral around each other as opposed to flying off tangentially. There are two main differences between 
our present simulations and the above referenced papers: First, we have a time integrated nonlinear response with a time lag, which means that the induced index inhomogeneity accumulates in time and becomes permanently imprinted within the medium. A consequence of this is that the field OAM need not be conserved under propagation once the MI and associated symmetry breaking leading to the formation of the fibers has occurred. This is the case since the propagating field now experiences an inhomogeneous medium in the form of the fibers, and one cannot appeal to conservation of field OAM to argue that the solitons and associated fibers need to travel tangentially. Second, the index changes involved in photopolymerization are large, $\simeq 0.04$, even by the standards of photorefractive effects, $\simeq 0.005$, so that forces that are known to arise between spatial solitons and give rise to spiraling are expected to be large. ${ }^{38,39}$ Furthermore, inspection of our numerical results for the fields propagating in the medium just following the formation of the individual fibers shows that the underlying solitons comprising the necklace beam are largely in-phase, not precisely due to the added noise, and based on this we expect attractive forces to arise between the spatial solitons. ${ }^{38,39}$ An example of this is shown in Figure 6, which for
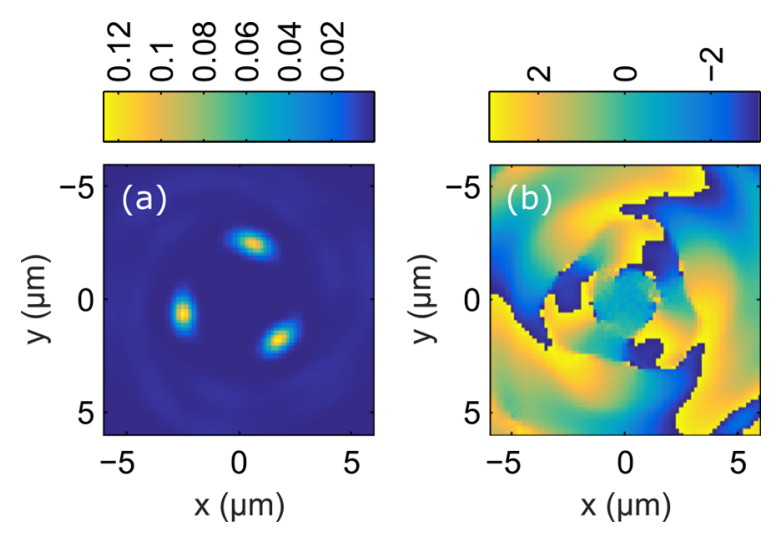

Figure 6. Spatial solitons. (a) Intensity profile of the three solitons and (b) the phase profile of the associated field.

$l=3$ and $z=100 \mu \mathrm{m}$ shows the intensity profile of the three solitons and its associated phase profile. The solitons and associated fibers generated in our photopolymerization simulations then exhibit helical trajectories due to the combined actions of the OAM supplied by the initial vortex, which on its own would lead to tangential motion with chirality determined by the sign of $l$, plus the attractive interactions between the solitons, which pulls the solitons and fibers towards each other and leads to the helical microfibers.

Finally, we mention that although the results of our model simulations agree qualitatively with the experiment in that they yield $|l|$ helical microfibers for an input vortex of topological charge $l$, the rotation rate with propagation distance of the helical fibers is consistently lower in the simulations, within a factor of 2 or so. We attribute this as likely due to the fact that our model uses a paraxial approximation to the electric field propagation which does not reflect the spiraling Poynting vector of the electromagnetic field. If the action of this spiraling Poynting vector was also incorporated into the material equation as an extra torque acting on the index change this could enhance the rotation rate, but this would require a vectorial treatment of the field propagation and a generalization of the material equation which is a study outside the scope of this present paper. On the other hand, for the beam sizes employed here corrections to the paraxial approximation are not expected to be large, meaning that our paraxial results should give the dominant contribution and the rotating Poynting vector is not essential to understanding the basic phenomenon.

\section{CONCLUSIONS}

We have both theoretically and experimentally investigated photopolymerization of UV curing resins with an optical vortex carrying OAM. The propagation of light in a photopolymer is described by the nonlinear wave equation for the slowly varying electric field due to the permanent refractive index change dependent on optical exposure. As a consequence of nonlinear self-focusing and self-trapping of the optical field, photopolymerization creates an optical soliton and an associated optical fiber with lengths up to a submillimeter scale. Due to modulation instability, the annular vortex beam breaks up into the $|l|$-solitons or microfibers, each of which exhibit helical trajectories with a chirality determined by the sign of $l$. These microfibers exhibit helical features that are indicative of the helical nature of the incident OAM field. This thus represents a new physical manifestation of the use of OAM light fields.

This result opens up a range of new possibilities for the use of structured light to photopolymerization and more broadly to applications such as writing novel mesoscopic structures in optical communications and for particle manipulation.

\section{MATERIALS AND METHODS}

Sample Preparation. We prepare a thin closed sample cell made of a vinyl sticker spacer ( $200 \mu \mathrm{m}$ in thickness) with a hole $(10 \mathrm{~mm}$ in diameter $)$, which is placed in between two type-1 borosilicate glass coverslips (VWR International, $150 \mu \mathrm{m}$ in thickness). $10 \mu l$ of a UV curing resin (Norland Optics, NOA63) is loaded onto the first coverslip in the middle of the vinyl sticker hole. The second coverslip is placed over the vinyl spacer and is sealed with a thin layer of nail polish to form an enclosed cell.

Experimental Setup. Figure 7 shows a schematic diagram of the experimental setup. A continuous wave (CW) diode laser with an emission wavelength of $405 \mathrm{~nm}$ (TOPTICA

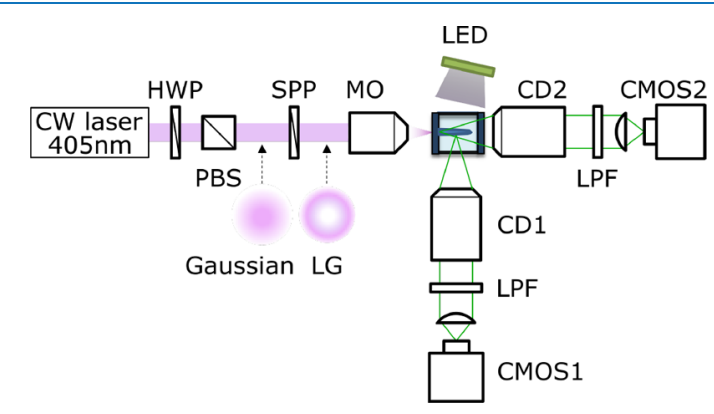

Figure 7. Schematic of experimental setup. A linearly polarized Gaussian beam is converted to an LG beam by a spiral phase plate (SPP). The vortex beam is focused by a microscope objective (MO) at the glass/resin interface to initiate the photopolymerization process, which is monitored by side- and axial-view cameras (CMOS1; CMOS2). Other labels denote the half-wave plate $(\mathrm{HWP})$, condenser lenses $(\mathrm{CD} 1, \times 50, \mathrm{NA}=0.55$; $\mathrm{CD} 2, \times 100$, NA $=0.70)$, long-pass filter (LPF, $\lambda>450 \mathrm{~nm}$ ), and light emitting diode (LED). 
Photonics AG, iBeam) is used to photopolymerize the UV curing resin in the sample cell. A linearly polarized beam after a polarizing beam splitter (PBS) cube is directed to a spiral phase plate (SPP, RCP Photonics, VPP-1c), where the Gaussian beam is converted to a Laguerre-Gaussian beam carrying topological charge in the range of $|l| \leq 4$. The vortex beam is focused by a high numerical aperture (NA) microscope objective $(\mathrm{MO}$, Olympus, $\times 60, \mathrm{NA}=1.1$ water immersion) to achieve an annular beam profile with a diameter of $1-2 \mu \mathrm{m}$ depending on $l$ (see Figure 2 inset panels and Supporting Information, Figure S3). The beam focus is carefully located at the glass/resin boundary in order to initiate the photopolymerization process on the glass surface of the sample cell. The laser power and the exposure time are fixed at $3 \mathrm{~mW}$ and $0.9 \mathrm{~s}$, respectively, unless stated otherwise.

Time-Lapse Imaging. Temporal evolution of the photopolymerization is recorded by two complementary metaloxide-semiconductor based cameras, for the side- and axialviews (CMOS1; CMOS2, Basler AG, acA1300-200um, 203 fps) at a frame rate of $100 \mathrm{fps}$. Time-lapse images recorded by the side-view camera visualize the growth of photopolymerized fibers and reveal their helical structure along the beam axis. The axial-view camera, on the other hand, can visualize the rotation of the fiber and its handedness.

SEM Imaging. After irradiation by the LG beam, the photopolymerized microstructure attached on the glass surface is rinsed with acetone to remove any uncured resins. A scanning electron microscope (SEM, JEOL, JSM-6010LA) is used to image the photopolymerized microstructure and quantify relevant microstructural features, such as helical pitch and dimensions of fabricated microfibers.

\section{ASSOCIATED CONTENT}

\section{S Supporting Information}

The following file is available free of charge. • supp.pdf: Supporting Information - Supplementary videos 1-5 The Supporting Information is available free of charge on the ACS Publications website at DOI: 10.1021/acsphotonics.8b00959.

Additional information as mentioned in the text (PDF). Supporting video 1 (AVI). Supporting video 2 (AVI). Supporting video 3 (AVI). Supporting video 4 (AVI). Supporting video 5 (AVI).

\section{AUTHOR INFORMATION}

\section{Corresponding Authors}

*E-mail: ya10@st-andrews.ac.uk; Phone: +44 (0)1334 461656.

*E-mail: omatsu@faculty.chiba-u.jp; Phone: +81 (0)43290 3477.

\section{ORCID $\odot$}

Yoshihiko Arita: 0000-0002-8704-545X

\section{Author Contributions}

J.L. and Y.A. contributed equally to this work. J.L., Y.A., and S.T. performed the experiments and data analysis. All authors contributed to the development and planning of the project, interpretation, and discussion of the data. Y.A., E.M.W., K.D., and T.O. wrote the manuscript. Y.A. and T.O. supervised the project.

\section{Notes}

The authors declare no competing financial interest.

\section{ACKNOWLEDGMENTS}

The authors acknowledge support from Grant-in-Aid for Scientific Research (Nos. JP 15H03571; JP 15K13373; JP 16K04971) from the Japan Society for the Promotion of Science (JSPS). We also acknowledge a financial support from JSPS KAKENHI Grants (JP 16H06507; JP 17K19070; JP 18H03884). P.P. is supported by the Air Force Office of Scientific Research (AFOSR) under Contract No. FA9550-161-0088 and by Office of Naval Research Multidisciplinary University Research Initiative (MURI) grant No N00014-171-2705. We thank the UK Engineering and Physical Sciences Research Council for support through Grant EP/P030017/1. We thank Dr. Graham D. Bruce, University of St Andrews, for useful discussions.

\section{REFERENCES}

(1) Kawata, S.; Sun, H. B.; Tanaka, T.; Takada, K. Finer features for functional microdevices. Nature 2001, 412, 697.

(2) Asavei, T.; Loke, V. L. Y.; Barbieri, M.; Nieminen, T. A.; Heckenberg, N. R.; Rubinsztein-Dunlop, H. Optical angular momentum transfer to microrotors fabricated by two-photon photopolymerization. New J. Phys. 2009, 11, 093021.

(3) Phillips, D. B.; Padgett, M. J.; Hanna, S.; Ho, Y. L. D.; Carberry, D. M.; Miles, M. J.; Simpson, S. H. Shape-induced force fields in optical trapping. Nat. Photonics 2014, 8, 400.

(4) Brasselet, E.; Malinauskas, M.; Žukauskas, A.; Juodkazis, S. Photopolymerized microscopic vortex beam generators: Precise delivery of optical orbital angular momentum. Appl. Phys. Lett. 2010, 97, 211108.

(5) Lightman, S.; Hurvitz, G.; Gvishi, R.; Arie, A. Miniature widespectrum mode sorter for vortex beams produced by $3 \mathrm{D}$ laser printing. Optica 2017, 4, 605.

(6) Paulsen, K. S.; Di Carlo, D.; Chung, A. J. Optofluidic fabrication for 3D-shaped particles. Nat. Commun. 2015, 6, 6976.

(7) Lee, H. J.; Jo, Y. R.; Kumar, S.; Yoo, S. J.; Kim, J. G.; Kim, Y. J.; Kim, B. J.; Lee, J. S. Close-packed polymer crystals from twomonomer-connected precursors. Nat. Commun. 2016, 7, 12803.

(8) Frisken, S. J. Light-induced optical wave-guide uptapers. Opt. Lett. 1993, 18, 1035.

(9) Kagami, M.; Yamashita, T.; Ito, H. Light-induced self-written three-dimensional optical waveguide. Appl. Phys. Lett. 2001, 79, 1079.

(10) Ježek, J.; Cižmár, T.; Neděla, V.; Zemánek, P. Formation of long and thin polymer fiber using nondiffracting beam. Opt. Express 2006, 14, 8506.

(11) Stankevicius, E.; Gertus, T.; Rutkauskas, M.; Gedvilas, M.; Raciukaitis, G.; Gadonas, R.; Smilgevicius, V.; Malinauskas, M. Fabrication of micro-tube arrays in photopolymer SZ2080 by using three different methods of a direct laser polymerization technique. J. Micromech. Microeng. 2012, 22, 065022.

(12) Malallah, R.; Cassidy, D.; Muniraj, I.; Ryle, J. P.; Healy, J. J.; Sheridan, J. T. Self-written waveguides in photopolymer. Appl. Opt. 2018, 57, E80.

(13) Allen, L.; Beijersbergen, M. W.; Spreeuw, R. J.; Woerdman, J. P. Orbital angular momentum of light and the transformation of Laguerre-Gaussian laser modes. Phys. Rev. A 1992, 45, 8185.

(14) Mazilu, M.; Arita, Y.; Vettenburg, T.; Auñón, J. M.; Wright, E. M.; Dholakia, K. Orbital-angular-momentum transfer to optically levitated microparticles in vacuum. Phys. Rev. A 2016, 94, 053821.

(15) Arita, Y.; Chen, M.; Wright, E. M.; Dholakia, K. Dynamics of a levitated microparticle in vacuum trapped by a perfect vortex beam: three-dimensional motion around a complex optical potential. J. Opt. Soc. Am. B 2017, 34, C14.

(16) Ramanathan, A.; Wright, K. C.; Muniz, S. R.; Zelan, M.; Hill, W. T.; Lobb, C. J.; Helmerson, K.; Phillips, W. D.; Campbell, G. K. Superflow in a toroidal Bose-Einstein condensate: an atom circuit with a tunable weak link. Phys. Rev. Lett. 2011, 106, 130401. 
(17) Toyoda, K.; Miyamoto, K.; Aoki, N.; Morita, R.; Omatsu, T. Using optical vortex to control the chirality of twisted metal nanostructures. Nano Lett. 2012, 12, 3645.

(18) Watabe, M.; Juman, G.; Miyamoto, K.; Omatsu, T. Light induced conch-shaped relief in an azo-polymer film. Sci. Rep. 2015, 4, 4281.

(19) Takahashi, F.; Takizawa, S.; Hidai, H.; Miyamoto, K.; Morita, R.; Omatsu, T. Optical vortex pulse illumination to create chiral monocrystalline silicon nanostructures. Phys. Status Solidi A 2016, 213, 1063.

(20) Leach, J.; Padgett, M. J.; Barnett, S. M.; Franke-Arnold, S.; Courtial, J. Measuring the orbital angular momentum of a single photon. Phys. Rev. Lett. 2002, 88, 257901.

(21) Fickler, R.; Lapkiewicz, R.; Plick, W. N.; Krenn, M.; Schaeff, C.; Ramelow, S.; Zeilinger, A. Quantum entanglement of high angular momenta. Science 2012, 338, 640.

(22) Xi, X. M.; Wong, G. K. L.; Frosz, M. H.; Babic, F.; Ahmed, G.; Jiang, X.; Euser, T. G.; Russell, P. S. J. Orbital-angular-momentumpreserving helical Bloch modes in twisted photonic crystal fiber. Optica 2014, 1, 165.

(23) Russell, P. S.; Beravat, R.; Wong, G. K. Helically twisted photonic crystal fibres. Philos. Trans. R. Soc. A 2017, 375, 20150440.

(24) Kewitsch, A. S.; Yariv, A. Self-focusing and self-trapping of optical beams upon photopolymerization. Opt. Lett. 1996, 21, 24.

(25) Padgett, M. J.; Miatto, F. M.; Lavery, M. P. J.; Zeilinger, A.; Boyd, R. W. Divergence of an orbital-angular-momentum-carrying beam upon propagation. New J. Phys. 2015, 17, 023011.

(26) Yariv, A.; Yeh, P. Photonics: Optical Electronics in Modern Communications, 6th ed.; Oxford University Press: New York; Oxford, 2007; p xii.

(27) Brüning, R.; Zhang, Y. W.; Mclaren, M.; Duparré, M.; Forbes, A. Overlap relation between free-space Laguerre Gaussian modes and step-index fiber modes. J. Opt. Soc. Am. A 2015, 32, 1678.

(28) Rekštyté, S.; Jonavičius, T.; Gailevicius, D.; Malinauskas, M.; Mizeikis, V.; Gamaly, E. G.; Juodkazis, S. Nanoscale precision of 3D polymerization via polarization control. Adv. Opt. Mater. 2016, 4, 1209.

(29) Chen, M.; Mazilu, M.; Arita, Y.; Wright, E. M.; Dholakia, K. Dynamics of microparticles trapped in a perfect vortex beam. Opt. Lett. 2013, 38, 4919.

(30) Chen, M.; Mazilu, M.; Arita, Y.; Wright, E. M.; Dholakia, K. Creating and probing of a perfect vortex in situ with an optically trapped particle. Opt. Rev. 2015, 22, 162.

(31) Kogelnik, H.; Li, T. Laser Beams and Resonators. Appl. Opt. 1966, 5, 1550.

(32) Soljačić, M.; Sears, S.; Segev, M. Self-trapping of "necklace" beams in self-focusing Kerr media. Phys. Rev. Lett. 1998, 81, 4851.

(33) Tikhonenko, V.; Christou, J.; Luther-Davies, B. Spiraling bright spatial solitons formed by the breakup of an optical vortex in a saturable self-focusing medium. J. Opt. Soc. Am. B 1995, 12, 2046.

(34) Tikhonenko, V.; Christou, J.; Luther-Davies, B. Three dimensional bright spatial soliton collision and fusion in a saturable nonlinear medium. Phys. Rev. Lett. 1996, 76, 2698.

(35) Firth, W. J.; Skryabin, D. V. Optical solitons carrying orbital angular momentum. Phys. Rev. Lett. 1997, 79, 2450.

(36) Walasik, W.; Silahli, S. Z.; Litchinitser, N. M. Dynamics of necklace beams in nonlinear colloidal suspensions. Sci. Rep. 2017, 7, 11709.

(37) Shih, M. F.; Jeng, C. C.; Sheu, F. W.; Lin, C. Y. Spatiotemporal optical modulation instability of coherent light in noninstantaneous nonlinear media. Phys. Rev. Lett. 2002, 88, 133902.

(38) Poladian, L.; Snyder, A. W.; Mitchell, D. J. Spiraling spatial solitons. Opt. Commun. 1991, 85, 59.

(39) Shih, M. F.; Segev, M.; Salamo, G. Three-dimensional spiraling of interacting spatial solitons. Phys. Rev. Lett. 1997, 78, 2551. 